\title{
Performance Comparison of Uncoded OFDM \& Uncoded Adaptive OFDM System Over AWGN Channel
}

\author{
Swati M. Kshirsagar ${ }^{1}$, A.N. Jadhav ${ }^{2}$ \\ ${ }^{12}$ Department of E \& TC, D.Y. Patil College of Engg. \& Tech., Kolhapur, Maharashtra, India.
}

\begin{abstract}
Adaptive OFDM (AOFDM) is the important approach to fourth generation of mobile communication. Adaptive modulating scheme is employed according to channel fading condition for improving the performance of OFDM. This gives improved data rate, spectral efficiency \& throughput. OFDM is flexible to adapt modulation schemes on subcarriers according instantaneous signal-to-noise ratio (SNR). In this paper, we compare Bit Error Rate (BER), Mean Square Error(MSE), Spectral Efficiency, Throughput performance of uncoded OFDM \& uncoded adaptive OFDM with BPSK, QPSK \& QAM modulation over AWGN channel.
\end{abstract}

Keywords - AOFDM, BER, MSE, Spectral Efficiency, Throughput

\section{Introduction}

Orthogonal Frequency Division Multiplexing (OFDM) is the most popular modulation technique for wireless communications. OFDM is a digital multicarrier modulation scheme which uses a large number of closely spaced orthogonal subcarriers [2]. OFDM signals are generated using the Fast Fourier transform. Each individual carrier, commonly called as subcarrier. Each subcarrier is modulated with a conventional modulation scheme at a low symbol rate, maintaining data rates similar to conventional single carrier modulation schemes in the same bandwidth. OFDM is a logical next step in broadband radio evolution. It is applied in IEEE standards like IEEE 802.11(Wi-Fi) and 802.16(WiMAX). OFDM is flexible to adapt modulation schemes on subcarriers according to instantaneous SNR. Adaptive OFDM (AOFDM) is the important approach to fourth generation of mobile communication. Adaptive modulation scheme is employed according to channel fading condition to improve OFDM performance. This improves data rate, spectral efficiency \& throughput.

In this paper, we analyze Bit Error Rate (BER), Mean Square Error (MSE), Spectral Efficiency, Throughput performance of uncoded OFDM \& uncoded adaptive OFDM with BPSK,QPSK \& QAM modulation over AWGN channel. This paper is organized as follows. We explain the concept of adaptive modulation in Section II. Section III gives system parameters. The performance of the proposed scheme in the presence of AWGN channel is explained in Section IV. Simulation results are given in Section V followed by concluding remarks.

II. System Model

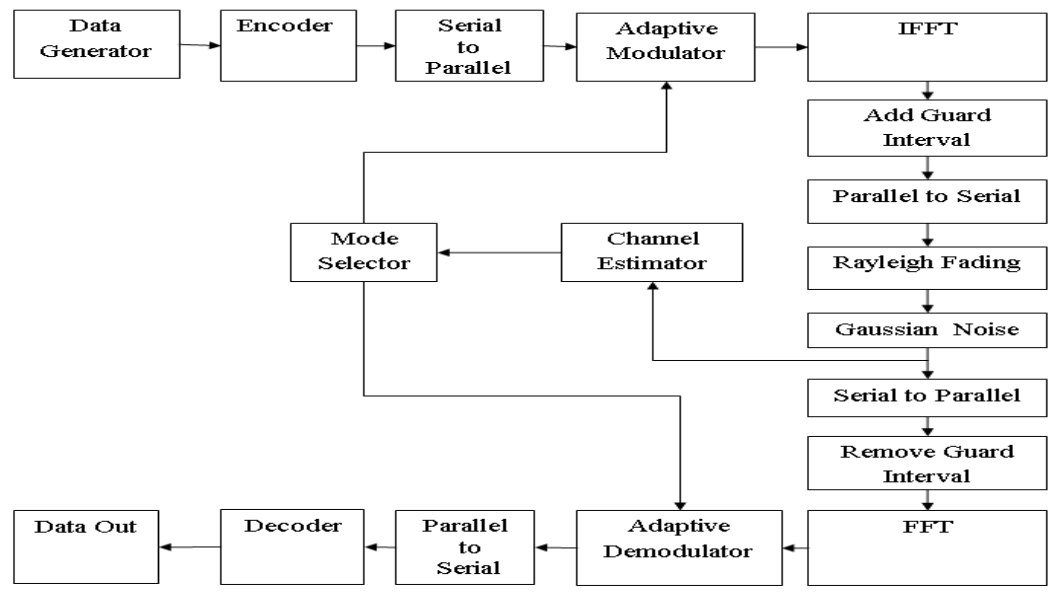

Figure 1. Adaptive OFDM system

The system model for Adaptive OFDM system is as shown in "Fig.1". The data is generated with the help of data generator. It is represented by a code word that consists of prescribed number code elements. The transmitter first converts this data from serial stream to parallel sets. Each set of data contains one symbol, $\mathrm{Si}$, for each subcarrier. For example, a set of four data would be [S0 S1 S2 S3]. The flexibility to rigorous channel 
conditions can be further improved if information about the channel is sent over a return channel. Based on this feedback information adaptive modulation \& channel coding may be applied across all subcarriers or individually to each subcarrier. An inverse Fourier transform (IFFT) converts the frequency domain data set into samples of the corresponding time domain representation. Specifically IFFT is useful for OFDM because it maintains orthogonality between subcarriers. Since the duration of each symbol is long it is feasible to insert a guard interval between the OFDM symbols. Thus eliminating intersymbol interference. Parallel to serial block creates the OFDM signal by sequentially outputting the time domain samples. At receiver guard interval is removed. FFT is applied to have frequency domain signals. Adaptive demodulator does the reverse of modulator.

The channel estimator is used to estimate the instantaneous SNR of the received signal. Based on the instantaneous SNR the best mode will be selected for next transmission frame. This task is done by the mode selector block [1].The channel estimation and mode selection are done at the receiver side and the information is sent to the transmitter using a feedback channel [4]. In this model the adaptation is done frame by frame. At the transmitter the adaptive modulator block consists of different modulators which are used to provide different modulation modes. The switching between these modulators will depend on the instantaneous SNR. This system model is used to describe three types of modulation schemes as BPSK, QPSK \& QAM.

\section{System Parameters}

Table1. System Parameters

\begin{tabular}{|c|c|}
\hline Parameter & Value \\
\hline IFFT size & 512 \\
\hline Number of subchannels N & 512 \\
\hline Number of subband & 32 \\
\hline Number of subcarriers per subband & 16 \\
\hline SNR & $0-30 \mathrm{~dB}$ \\
\hline Guard interval N/4 & 128 \\
\hline Pilot interval & 8 \\
\hline Modulation scheme & BPSK,QPSK, QAM \\
\hline Channel length L & 16 \\
\hline Number of pilots(P= N/8) & 64 \\
\hline
\end{tabular}

OFDM system parameters considered here to analyze the Bit Error Rate (BER),Mean Square Error(MSE), Spectral Efficiency, Throughput performance of uncoded OFDM \& uncoded adaptive OFDM with BPSK,QPSK \& QAM modulation over AWGN channel are mentioned in Table1.

\section{Results And Discussions}

Here we analyzed the the Bit Error Rate (BER),Mean Square Error, Spectral Efficiency, Throughput performance of uncoded OFDM \& uncoded adaptive OFDM with BPSK, QPSK \& QAM modulation over AWGN channel. Here we have used Cyclic coding. "Fig.2" \& "Fig.3" shows BER performance of uncoded OFDM \& uncoded adaptive OFDM respectively. It is observed from "Fig.2" that BER of uncoded OFDM decreases as SNR value increases also from "Fig.3"for SNR is in between $0 \mathrm{~dB}-3 \mathrm{~dB}$, highest modulation scheme i.e.64QAM is used. For SNR is between $3 \mathrm{~dB}-6 \mathrm{~dB}, 32 \mathrm{QAM}$ is used. When SNR is in between $6 \mathrm{~dB}$ $9 \mathrm{~dB}$ modulation scheme 16 QAM is used. For SNR in between $9 \mathrm{~dB}-12 \mathrm{~dB}, 8 \mathrm{QAM}$ is used. For SNR is between $12 \mathrm{~dB}-15 \mathrm{~dB}$ lower modulation scheme i.e. QPSK is used \& for SNR is from $12 \mathrm{~dB}-18 \mathrm{~dB}$ lowest modulation scheme i.e. BPSK is used. For value of SNR above $18 \mathrm{~dB}$ signal is not transmitted. Lower modulation schemes has better BER performance as compared to higher one for higher SNR value. Thus adaptation is achieved on the basis of instantaneous SNR. Thus BER performance of uncoded adaptive OFDM is better than uncoded OFDM. 


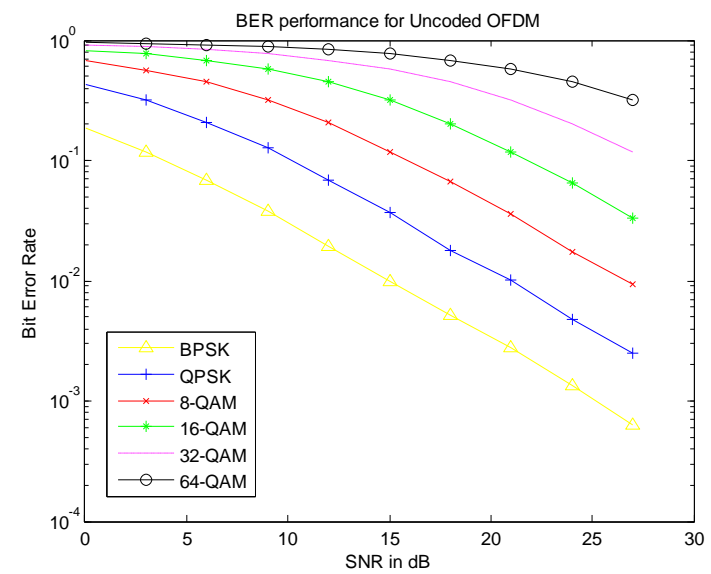

Figure 2. BER Performance of Uncoded OFDM with BPSK,QPSK,QAM

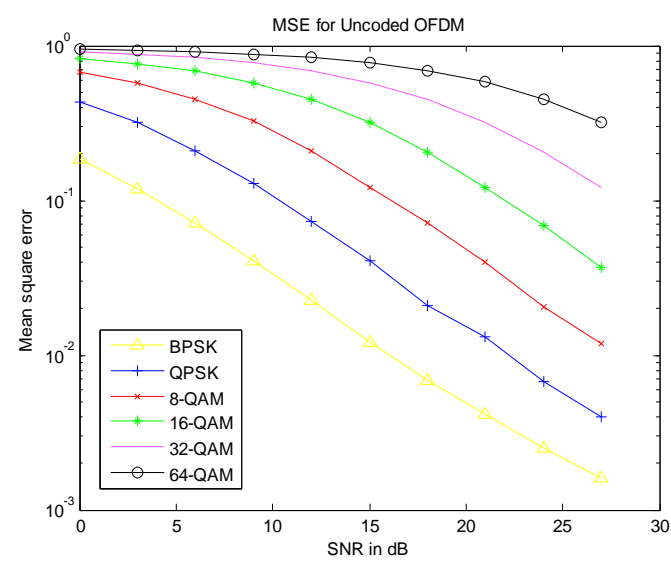

Figure 4. Mean Square Error of Uncoded OFDM with BPSK, QPSK,QAM

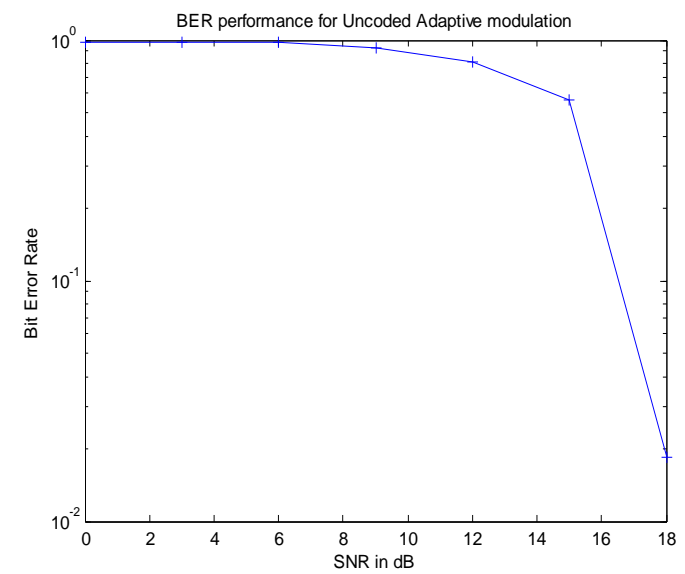

Figure 3. BER Performance of Uncoded AOFDM with BPSK, QPSK,QAM

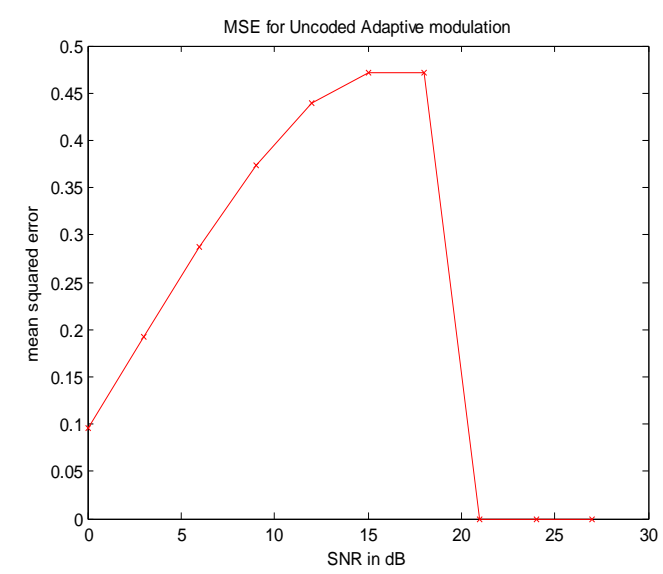

Figure 5. Mean Square Error of Uncoded AOFDM with BPSK, QPSK,QAM

It is observed from "Fig.4" that Mean Square Error (MSE) of uncoded OFDM decrease as SNR increases. From "Fig.5" Mean Square Error (MSE) of uncoded adaptive OFDM with BPSK,QPSK,QAM increases from 0.1 to 0.47 for SNR value from $0 \mathrm{~dB}$ to $18 \mathrm{~dB}$. After that it decreases \& becomes 0 at \& above SNR $21 \mathrm{~dB}$ due to no transmission.

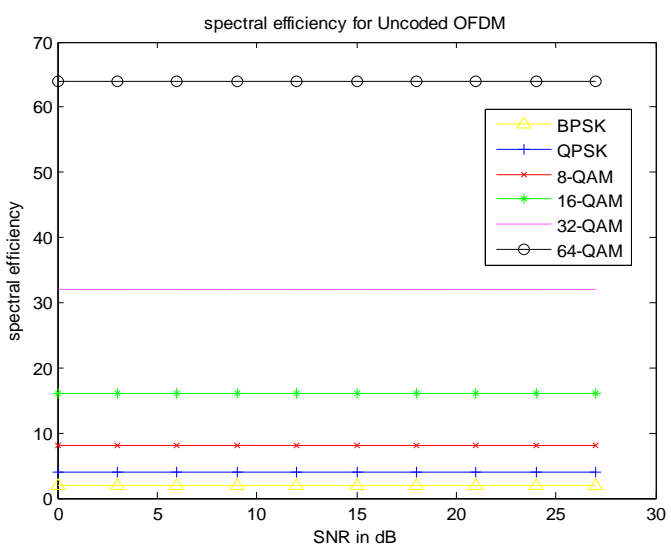

Figure 6. Spectral efficiency of Uncoded OFDM with BPSK, QPSK,QAM

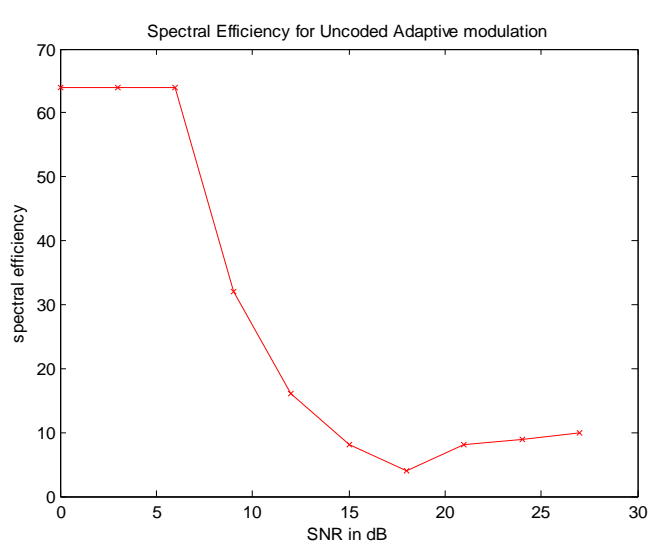

Figure 7. Spectral efficiency of Uncoded AOFDM with BPSK, QPSK,QAM

Spectral efficiency of uncoded OFDM \& coded adaptive OFDM with BPSK,QPSK,QAM is shown in "Fig.6" \& "Fig.7" respectively . "Fig.6" shows that Spectral efficiency of BPSK, QPSK, 8QAM, 16 QAM, 32 QAM \& 64 QAM is $2 \%, 4 \%, 8 \%, 16 \%, 32 \%, 64 \%$ respectively. "Fig.7" shows that for SNR OdB to $6 \mathrm{~dB}$ uncoded AOFDM shows better Spectral efficiency as about 64\% .As SNR is still increasing accordingly Spectral efficiency decreases . 


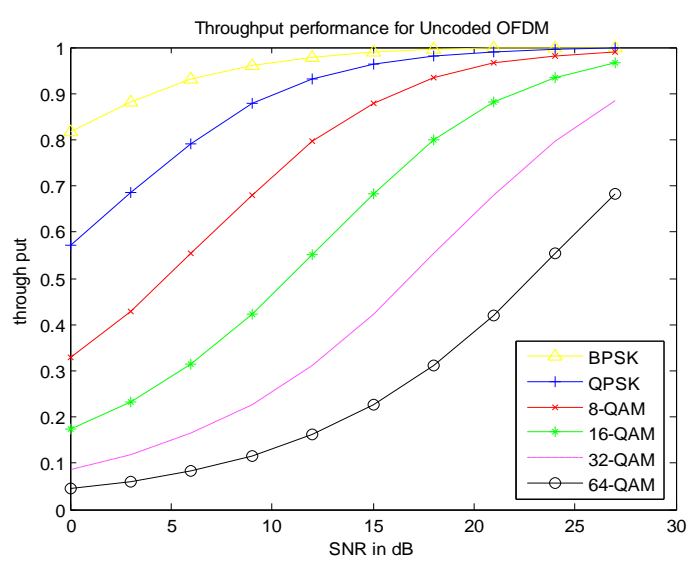

Figure 8. Throughput of Uncoded OFDM with BPSK,QPSK, QAM

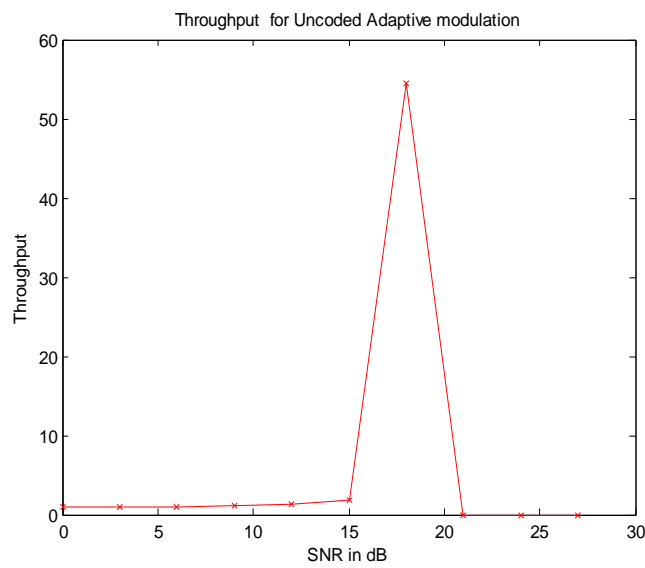

Figure 9. Throughput of Uncoded AOFDM with BPSK,QPSK, QAM

Throughput of uncoded OFDM \& uncoded adaptive OFDM with BPSK,QPSK,QAM is shown in "Fig.8" \& "Fig.9" respectively . From "Fig.8" as SNR increases throughput of each modulation scheme increases accordingly. Lower modulation scheme has better Throughput. For uncoded AOFDM SNR upto $12 \mathrm{~dB}$ due to higher level modulation schemes Throughput is less. As SNR increases above 12dB lower level modulation schemes as QPSK \& BPSK are employed so Throughput increases. Throughput becomes maximum as 54.47 for SNR value $18 \mathrm{~dB}$. After SNR above $18 \mathrm{~dB}$ it decreases. For SNR values in between $21 \mathrm{~dB}$ to $27 \mathrm{~dB}$ Throughput is minimum i.e. 0 due to no transmission.

\section{Conclusions}

In this paper, we have evaluated Bit Error Rate (BER), Mean Square Error (MSE), Spectral Efficiency, Throughput performance of uncoded OFDM \& uncoded adaptive OFDM with BPSK,QPSK \& QAM modulation over AWGN channel. In uncoded adaptive OFDM according to instantaneous SNR suitable modulation scheme is employed. BER performance of uncoded adaptive OFDM is better than uncoded OFDM. MSE of uncoded adaptive OFDM increases from 0.1 to 0.47 for SNR value from OdB to $18 \mathrm{~dB}$. After that it decreases $\&$ becomes 0 at $\&$ above SNR $21 \mathrm{~dB}$ due to no transmission. For SNR 0dB to 6 dB uncoded adaptive OFDM shows better Spectral efficiency as about 64\%.As SNR is still increasing accordingly Spectral efficiency decreases .For uncoded AOFDM SNR upto 12dB Throughput is less. As SNR increases above 12dB Throughput increases. Throughput becomes maximum as 54.47 for SNR value $18 \mathrm{~dB}$. After SNR above $18 \mathrm{~dB}$ it decreases. For SNR values in between $21 \mathrm{~dB}$ to $27 \mathrm{~dB}$ Throughput is minimum i.e. 0.Thus Adaptive modulation achieves a good tradeoff between spectral efficiency and overall BER .

\section{References}

[1] A.Sohail and M.N.Jafri, "Adaptive OFDM over Frequency Selective and Fast Fading Channel Using Blockwise Bit Loading Algorithm”, IEEE International Conferenece on Wireless and Optical Communication Networks,pp. 1-4, July 2007.

[2] A.Cyzlwik, "Adaptive OFDM for wideband radio channels", Global Telecommunications Conference, vol 1, pp713-718, Nov 1996.

[3] X She ,Z.Zhang,S.Zhou,Yan Yao, “Adaptive Turbo Coded Modulation for OFDM Transmissions ”, Proceedings of ICCT.pp.1491$1495,2003$.

[4] K.M.Hadi , R.Tripathi and K.Kant, “ Performance of Adaptive Modulation in Multipath Fading Channel”,The 8th International Conference on Advanced Communication Technology, ICACT, vol. 2, pp.1277- 1282, 20-22 February 2006.

[5] L. Khalid and A. Anpalagan, "Threshold-Based Adaptive Modulation with Adaptive Subcarrier Allocation in OFCDM-Based 4G Wireless Systems," IEEE VehicularTechnology Conference, pp.1-6, Sept. 2006.

[6] S.Sampei and H.Harada, "System Design Issues and Performance Evaluations for Adaptive Modulation in New Wireless Access Systems", Proceedings of the IEEE, Vol .95, no.12, pp. 2456-2471,Dec 2007.

[7] B. Le Flock, M. Alard, and C. Berrou, "Coded orthogonal frequency division multiplex," Proceedings of the IEEE, Vol. 83, no. 6, June 1995.

[8] T.S.Rappaport, Wireless Communications Principles \& Practice ( II ${ }^{\text {nd }}$ Edition .Pentice-Hall, January 2002).

[9] J.G.Prokis,Digital Communications (Mc-Graw Hill, New York NY, IV ${ }^{\text {th }}$ Edition ,2000). 